\title{
Metabolic Syndrome in IgA Glomerulonephritis
}

\author{
Kati Kaartinen ${ }^{a}$ Jaana Syrjänen ${ }^{a, c}$ Ilkka Pörsti ${ }^{a, c}$ Aimo Harmoinen ${ }^{d}$ \\ Heini Huhtalab Jukka Mustonen ${ }^{a, c}$ \\ Schools of a Medicine and ${ }^{b}$ Health Sciences, University of Tampere, and ${ }^{c}$ Department of \\ Internal Medicine, Tampere University Hospital, Tampere, and ${ }^{\mathrm{d}}$ Department of Laboratory \\ Medicine, Central Hospital of Savonlinna, Savonlinna, Finland
}

\section{Key Words}

Creatinine $\cdot$ Cystatin-C $\cdot$ Estimated glomerular filtration rate $\cdot$ IgA glomerulonephritis .

Metabolic syndrome

\begin{abstract}
Background/Aims: Metabolic syndrome (MetS) may have an independent impact on the development of chronic kidney disease. This study examines the prevalence of MetS in subjects with IgA glomerulonephritis (IgAGN) and its impact on disease progression in a retrospective fashion. Patients and Methods: Altogether, 174 subjects (104 males) were examined 11 years (first visit) after IgAGN diagnosis and again after 16 years (second visit; 144 subjects responded). Different glomerular filtration markers were utilized. The MetS criteria by Alberti et al. [Circulation 2009;120:1640-1645] were applied, in which the presence of any three of five risk factors (elevated waist circumference, triglycerides, glucose, existence of hypertension, or reduced high-density lipoprotein cholesterol) constitutes the diagnosis. Results: The prevalence of MetS at the first visit was 39\%, corresponding to that of the general Finnish population. In univariate analyses, MetS was significantly associated with the progression of IgAGN at the second visit. However, in multivariate analyses, the existence of MetS was not a significant prognostic determinant. Conclusion: The number of subjects with MetS among IgAGN patients and the general population is equal in Finland. MetS does not seem to be an independent prognostic variable.


Kaartinen et al.: Metabolic Syndrome in IgA Glomerulonephritis

\section{Introduction}

Metabolic syndrome (MetS) is characterized by a cluster of atherosclerotic risk factors including obesity, hypertension, insulin resistance, and dyslipidemia [1]. In patients with chronic kidney disease (CKD), the prevalence of MetS has varied between 14 and 31\% [2-5]. Some follow-up studies have suggested that MetS has an independent adverse impact on the development of CKD [6, 7]. MetS criteria may significantly influence the outcome of the calculated risk of developing CKD [8]. In addition, it is unclear which glomerular filtration rate (GFR) estimate is the most useful when investigating MetS, as different results may be obtained with different GFR methods [9].

IgA glomerulonephritis (IgAGN) is the most common primary form of glomerulonephritis. The most consistently reported factors for poor prognosis have been hypertension, the amount of proteinuria, and kidney insufficiency [10]. Recently, we have reported that insulin resistance was linked with poor outcome in IgAGN; however, its independent role was not clear [11]. Only one report has assessed the role of MetS in IgAGN, and the results of this cross-sectional study suggested that hypertension was the only significant variable affecting serum creatinine [12]. The purpose of this study was to investigate the prevalence of MetS and its impact on the progression in a cohort of biopsy-proven IgAGN patients in a follow-up study.

\section{Patients and Methods}

\section{Patients}

The original population consisted of patients living in the Pirkanmaa Health District in Finland (population about 440,000) in whom IgAGN was diagnosed during the period between January 1, 1980, and December 31, 1990 (223 patients) [13]. The definition of IgAGN was based on immunofluorescence microscopy showing that IgA was the sole or main glomerular finding on renal biopsy. The detailed description of histology has been reported elsewhere [14]. From the original retrospective group, a cohort was invited to attend two physician appointments separated by 6 years. The study flow, the number of patients, and gender distribution are depicted in figure 1.

At the first visit, median age was 48.5 years (range 17-85) and median time from renal biopsy 11 years (range 6-17). All patients had been diagnosed at least 5 years and 63\% at least 10 years before the first visit. Median time from the first signs of IgAGN (episode of macroscopic hematuria, discovery of microscopic hematuria or proteinuria, or renal insufficiency) was 14 years (range $7-57$ ). Age at diagnosis was $\leq 40$ years in $49 \%$ of the patients. At the second visit, median age was 54 years (range 23-90) and time from biopsy 16 years (range 11-24). A total of $97 \%$ had been diagnosed at least 10 years before that visit. Median time from the first signs of IgAGN was 19 years (range 12-64) at that time.

Eleven patients presented with some manifestations of Henoch-Schönlein purpura at renal diagnosis. Altogether, end-stage renal disease (ESRD) had developed in 10 of 174 (6\%) patients by the first visit. As features of MetS are frequent in ESRD and may thus possess a bias, these patients were excluded from the subsequent analyses.

\section{Clinical Data}

MetS was defined according to the latest criteria by a joint interim statement of several organizations with waist circumference thresholds recommended for Caucasian populations ( $\geq 94 \mathrm{~cm}$ for males and $\geq 80 \mathrm{~cm}$ for females) [1]. The presence of any three of five risk factors constituted a diagnosis of MetS. The criteria for hypertension and elevated fasting glucose 
Kaartinen et al.: Metabolic Syndrome in IgA Glomerulonephritis

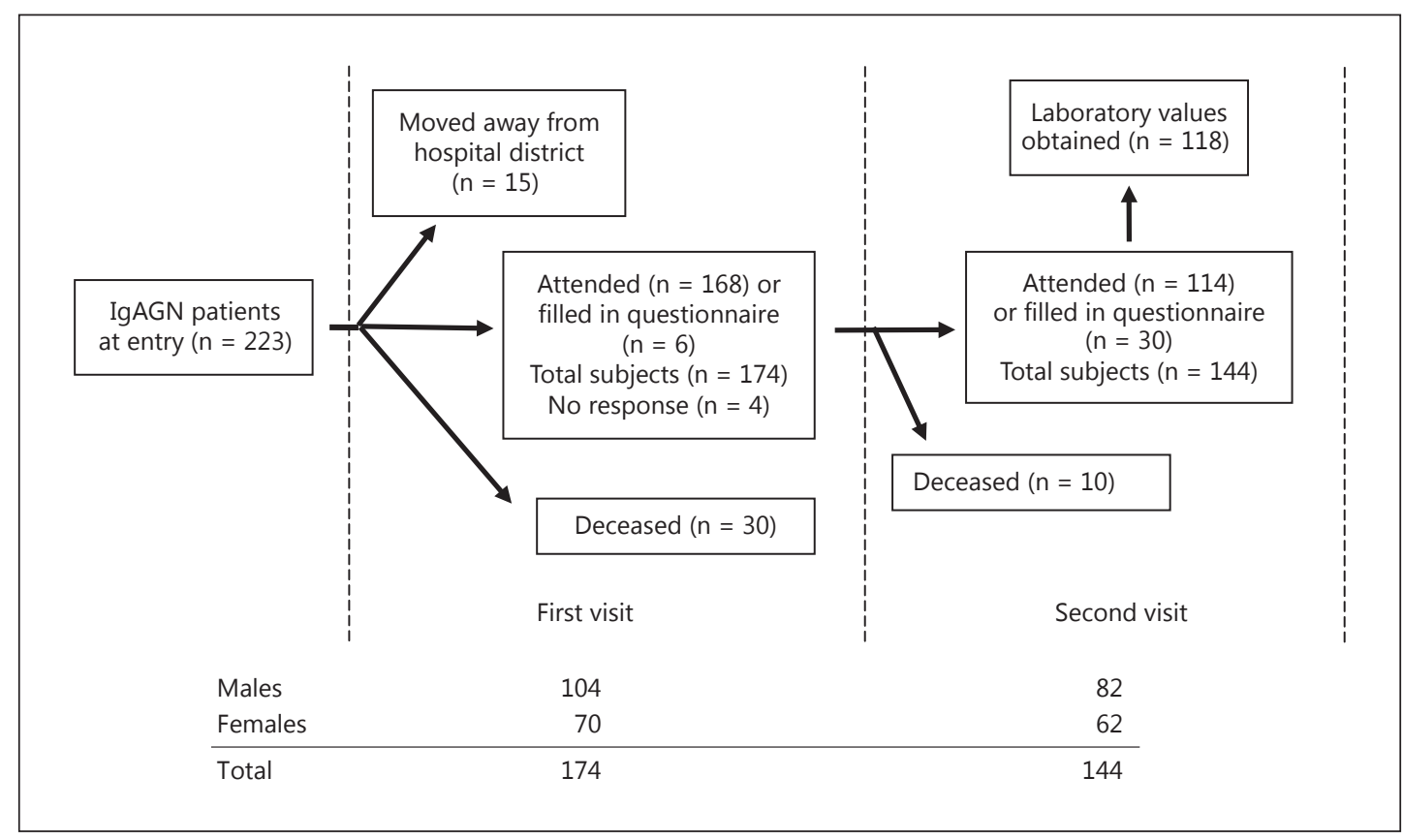

Fig. 1. Description of the study flow, the number of patients, and the distribution of gender.

Table 1. Criteria for the clinical diagnosis of MetS by Alberti et al. [1]

\begin{tabular}{ll}
\hline Measure & $\begin{array}{l}\text { Categorical } \\
\text { cut points }\end{array}$ \\
\hline Elevated waist circumference, cm & $\geq 94$ \\
$\quad$ Males & $\geq 80$ \\
$\quad$ Females & $\geq 1.7$ \\
Elevated triglycerides (or drug treatment for elevated triglycerides), mmol/l & $<1.0$ \\
Reduced HDL cholesterol (or drug treatment for reduced HDL cholesterol), mmol/l & $<1.3$ \\
$\quad$ Males & $\geq 130$ \\
$\quad$ Females & $\geq 85$ \\
$\quad$ Slevated blood pressure (or antihypertensive drug treatment), mm Hg & $\geq 5.6$ \\
$\quad$ Diastolic & \\
Elevated fasting glucose (or drug treatment of elevated glucose), mmol/l & \\
\hline
\end{tabular}

The presence of any 3 of 5 risk factors constitutes a diagnosis of MetS. Waist circumference thresholds for abdominal obesity are those used for Caucasian populations. HDL = High-density lipoprotein.

were according to the definition of MetS (table 1). The median values for systolic blood pressure were $140 \mathrm{~mm} \mathrm{Hg}$ (range 104-190) at the first visit and $142 \mathrm{~mm} \mathrm{Hg}$ (range 90-224) at the second visit. Diastolic blood pressure values were $90 \mathrm{~mm} \mathrm{Hg}$ (range 60-118) and 90 $\mathrm{mm} \mathrm{Hg}$ (range 52-120), respectively. The number of diabetic subjects was 21 by the second visit. Medians for the body mass index were 26 (range 18-45) and 27 (range 19-43), and for the amounts of proteinuria $0.31 \mathrm{~g}$ (range $0.08-7.34$ ) and $0.19 \mathrm{~g}$ (range 0.03-9.13), respectively. 
Progressive IgAGN at the second visit was defined as an elevation of serum cystatin-C or creatinine above the normal level and over $20 \%$ elevation from the value at the first visit. Progression was analogously defined as a reduction in estimated GFR (eGFR was calculated by the MDRD equation) below the normal level and an over $20 \%$ reduction from the value at the first visit. The $20 \%$ rule was added in order to avoid misclassification of patients with elevated cystatin-C or creatinine or reduced eGFR but still a stable disease. We have previously used the same criteria for the progression of IgAGN $[11,13]$.

\section{Study Protocols and Ethical Aspects}

The baseline clinical data of the patients were collected from medical records. The followup included two clinical visits separated by approximately 6 years. Data on medication, concurrent diseases, smoking and alcohol drinking habits as well as anthropometric measures, blood pressure, and laboratory variables were recorded during the visits. Causes of death were confirmed from the patient files or from the death certificates kept by Statistics Finland. The follow-up ended at the second visit or at the latest available check obtained from the medical records or the questionnaires sent to the patients not attending the visits. The study protocol was approved by the Ethics Committee of Tampere University Hospital, and the study was carried out according to the provisions of the Declaration of Helsinki.

\section{Laboratory Measurements}

Laboratory variables were analyzed using the fasting serum, spot, and collection urine samples utilizing in-house routine analytical methods of the laboratory of Tampere University Hospital. Serum cystatin-C was analyzed using the immunoturbidometry method with Cobas Mira S (F. Hoffmann-La Roche, Basel, Switzerland), and values were considered normal if $<1.2 \mathrm{mg} / \mathrm{l}$ ( $\leq 50$ years) or $<1.4 \mathrm{mg} / \mathrm{l}$ ( $>50$ years). A six-variable eGFR [15] was calculated and considered normal if $\geq 60 \mathrm{ml} / \mathrm{min} / 1.73 \mathrm{~m}^{2}$. Serum creatinine was considered normal if $<105 \mu \mathrm{mol} / \mathrm{l}$ for males and $<95 \mu \mathrm{mol} / \mathrm{l}$ for females.

\section{Statistical Analyses}

The SPSS for Windows 17.0 package was used for statistical analyses (SPSS Inc., Chicago, Ill., USA), and a two-sided p value $<0.05$ was taken as the level for statistical significance. Associations between categorical variables and continuous nonnormally distributed variables were calculated using the Mann-Whitney U test. Associations between categorical variables and continuous, normally distributed variables were calculated using Student's $t$ test. Relationships between two nonnormally distributed paired groups were analyzed by the Wilcoxon test. Odds ratios (OR) and 95\% confidence intervals (CI) were computed using logistic regression analyses (enter method). Goodness of fit of the models was tested with the Hosmer-Lemeshow test.

\section{Results}

\section{Kidney Function and the Progression of IgAGN}

The results were essentially unchanged with or without inclusion of secondary forms of IgAGN (data not shown). Kidney function at the visits is presented in table 2. At the second visit, IgAGN was classified as progressive in 20 of 114 (18\%) patients using cystatin-C based evaluation, in 27 of 152 (18\%) using serum creatinine, and in 23 of $117(20 \%)$ patients using eGFR. Angiotensin-converting enzyme (ACE) medication was used in 26.2 and $29.5 \%$ of the patients at the first and second visit, respectively, and the change was statistically significant. Angiotensin receptor blockers were not available at the first visit and 9.6\% used them at the second visit, the change was not statistically significant. 
Table 2. Median (range) values for kidney function during the visits

\begin{tabular}{llll}
\hline Variable & First visit $(\mathrm{n}=154-164)$ & Second visit $(\mathrm{n}=115-164)$ & $\mathrm{p}$ value \\
\hline S-cystatin-C, $\mathrm{mg} / \mathrm{l}$ & $0.76(0.44-2.45)$ & $1.1(0.59-2.93)$ & $<0.001$ \\
S-creatinine, $\mu \mathrm{mol} / \mathrm{l}$ & $81(42-322)$ & $91(45-888)$ & $<0.001$ \\
eGFR, $\mathrm{ml} / \mathrm{min} / 1.73 \mathrm{~m}^{2}$ & $81(18-165)$ & $73(12-128)$ & $<0.001$ \\
\hline
\end{tabular}

The Wilcoxon test was used for the comparison of two paired groups.

Table 3. Percentage of IgAGN patients having MetS and its different components at the first visit

\begin{tabular}{llcc}
\hline Measure & All (n = 164) & Males (n=98) & Females (n=66) \\
\hline MetS & $39 \%$ & $43 \%$ & $36 \%$ \\
Elevated waist circumference & $52 \%$ & $50 \%$ & $53 \%$ \\
Elevated triglycerides (or drug treatment) & $33 \%$ & $42 \%$ & $23 \%$ \\
Reduced HDL cholesterol (or drug treatment) & $41 \%$ & $44 \%$ & $37 \%$ \\
Elevated blood pressure (or drug treatment) & $66 \%$ & $71 \%$ & $60 \%$ \\
Elevated fasting glucose (or drug treatment) & $17 \%$ & $24 \%$ & $7 \%$ \\
\hline
\end{tabular}

Table 4. Clinical and biochemical characteristics of patients with and without MetS at the first visit

\begin{tabular}{lccc}
\hline Measure & Patients without MetS (n=95) & Patients with MetS (n=60) & $\mathrm{p}$ value \\
\hline Age, years & $43(17-81)$ & $56(28-85)$ & $<0.001$ \\
Weight, kg & $72(42-110)$ & $88(65-145)$ & $<0.001$ \\
Systolic blood pressure, mm Hg & $135(104-190)$ & $154(122-190)$ & $<0.001$ \\
Diastolic blood pressure, mm Hg & $88(65-118)$ & $90(60-110)$ & 0.03 \\
ACE medication, \% & 21 & 38 & 0.02 \\
ARBs, \% & 0 & 0 & n.s. \\
Cystatin-C, mg/l & $0.7(0.4-5.1)$ & $0.8(0.4-1.8)$ & 0.003 \\
S-creatinine, mmol/l & $79(44-888)$ & $94(42-238)$ & 0.003 \\
eGFR, ml/min/1.73 m ${ }^{2}$ & $89(5-165)$ & $69(26-164)$ & 0.003 \\
Proteinuria, g/24 h & $0.25(0.08-5.38)$ & $0.45(0.09-7.34)$ & 0.01 \\
Cholesterol, mmol/l & $5.2(2.8-8.4)$ & $5.5(3.8-7.6)$ & 0.003 \\
HDL cholesterol, mmol/l & $1.31(0.62-2.46)$ & $1.00(0.64-1.58)$ & $<0.001$ \\
Triglycerides, mmol/l & $1.04(0.42-3.95)$ & $1.85(0.77-7.5)$ & $<0.001$ \\
LDL cholesterol, mmol/l & $3.2(1.3-5.7)$ & $3.5(2.2-5.2)$ & 0.02 \\
\hline
\end{tabular}

Data are presented as median (range), unless otherwise indicated. The Mann-Whitney U test or Student's $\mathrm{t}$ test were used, depending on the distribution of the variable. n.s. = Not significant; $\mathrm{ARBs}=$ angiotensin receptor blockers; HDL = high-density lipoprotein; LDL = low-density lipoprotein.

Associations of MetS at the First Visit with the Progression of IgAGN at the Second Visit

Altogether, the prevalence of MetS was 39\% with preponderance in males (43\%) and a somewhat lower frequency in females (36\%). The most prevalent feature of MetS was hypertension, followed by elevated waist circumference in both sexes (table 3).

Having MetS at the first visit was associated with worse kidney function at the second visit, whether it was assessed using cystatin-C $(p<0.001)$, creatinine $(p=0.001)$, or eGFR $(\mathrm{p}=0.005)$. Univariate analyses showed that MetS was associated with progression with 
Table 5. Associations between MetS and known risk factors for progressive IgAGN at the first visit and the progression of IgAGN observed at the second visit

Kaartinen et al.: Metabolic Syndrome in IgA Glomerulonephritis

\begin{tabular}{llll}
\hline Variable & OR & $95 \%$ CI & p value \\
\hline $\begin{array}{l}\text { Cystatin-C-based estimate } \\
\quad \text { MetS }\end{array}$ & 1.9 & $0.6-6.0$ & 0.258 \\
$\quad$ 24-hour protein excretion & 2.4 & $1.3-4.3$ & 0.004 \\
$\quad$ Age & 1.0 & $0.9-1.1$ & 0.08 \\
$\quad$ ACE medication & 0.5 & $0.2-1.6$ & 0.264 \\
Creatinine-based estimate & & & \\
$\quad$ MetS & 1.4 & $0.5-4.0$ & 0.505 \\
$\quad$ 24-hour protein excretion & 2.4 & $1.4-4.2$ & 0.003 \\
$\quad$ Age & 1.1 & $1.0-1.1$ & 0.011 \\
$\quad$ ACE medication & 0.4 & $0.1-0.9$ & 0.049 \\
eGFR-based estimate & & & \\
$\quad$ MetS & 1.9 & $0.6-6.1$ & 0.273 \\
24-hour protein excretion & 2.7 & $1.4-5.2$ & 0.003 \\
Age & 1.1 & $1.0-1.1$ & 0.005 \\
$\quad$ ACE medication & 0.3 & $0.1-0.9$ & 0.029 \\
\hline
\end{tabular}

Multivariate analysis with all shown variables in the model. The Hosmer-Lemeshow test for goodness of fit had a p value of 0.139 for cystatin-C-based estimate, of 0.899 for creatinine-based estimate, and of 0.869 for eGFR-based estimate.

nearly all filtration markers: cystatin-C (OR 4.6, 95\% CI 1.4-14.8, p = 0.012), creatinine (OR 2.4, 95\% CI 0.9-6.0, $\mathrm{p}=0.057$ ), and eGFR (OR 3.0, 95\% CI 1.1-8.3, $\mathrm{p}=0.039$ ). Univariate analyses of all components of MetS showed that only hypertension was associated with progression with all filtration markers (data not shown).

Patients with and without MetS had significant differences in clinical and biochemical variables at the first visit (table 4). However, only age, systolic blood pressure, use of ACE medication, and proteinuria were significant determinants in univariate analyses (data not shown) and were therefore included in multivariate analyses. In multivariate analysis, MetS was not a significant determinant of progression (table 5). Twenty-four-hour protein excretion was, as expected, a significant prognostic factor. Age and use of ACE medication were significant determinants when progression was based on serum creatinine or MDRD estimates. As hypertension is included in the criteria for MetS, it was not considered separately in the multivariate model.

\section{Discussion}

To our knowledge, this is the first report assessing the influence of MetS on the progression of IgAGN. Univariate analyses suggested that MetS negatively affects the progression of IgAGN. However, in combination with other known prognostic factors, it was not a significant determinant. Among the different components of MetS, hypertension was the most prevalent in almost $70 \%$ of the patients and it seemed to be the most important feature of MetS. Cystatin$\mathrm{C}$ concentration may be increased in MetS independently of changes in creatinine clearance, but this could also reflect slight GFR impairment not detectable by serum creatinine [9]. Therefore, we used three parallel filtration markers, and the majority of the analyses yielded similar results.

The population described here comprised IgAGN patients with conditions ranging from normal renal function to severe kidney dysfunction, excluding ESRD before the first visit. 
Four patients developed ESRD between the visits, but most patients had normal kidney function even at the end of the study. The prevalence of MetS in this patient group was higher than expected from previous reports from kidney patients. The reported prevalence ranged between 10.7 and $30.5 \%$, although a subgroup of renal transplant recipients with a failing graft had a MetS prevalence of 52\% [2-5]. In Finland, the prevalence of MetS ranged between 30.2 and $47.8 \%$ in a population-based study using the most recent criteria for 30-79-year-old subjects and was strongly dependent on the criteria and sex [16]. The median age of our patients at the first visit was 49 years making the prevalence of MetS in our IgAGN patients correspond to that in the general population in Finland.

We chose to utilize the most recent criteria of MetS, as these contain the most updated information. Hypertension is probably the most important single feature of MetS, at least in renal patients. Among variables comprising the criteria for MetS, hypertension was reported to be the only variable that affected serum creatinine levels in a cross-sectional case-control study with 60 IgAGN patients, and our results are in line with that [12].

Visceral obesity and the subsequent insulin resistance, inappropriate activation of the renin-angiotensin system, and increased oxidative stress have been suggested as possible links between MetSand renal riskleading to glomerular hypertension, endothelial dysfunction, vasoconstriction, extracellular matrix expansion, and aldosterone excess $[17,18]$. Insulin resistance is a major element of MetS, and the results of our former study suggested that insulin resistance negatively influences prognosis in IgAGN along with hypertriglyceridemia $[11,13]$. However, in a multivariate setting together with known risk factors for progression, MetS did not seem to have an independent role despite a cluster of potentially harmful variables. As hypertension was the only significant prognostic variable among different components of MetS, its importance weakened when combined with less important variables. It must be stated that in the current analysis, the heaviest progressors were excluded, as they had already achieved ESRD before the first visit. In addition, the time scale was different from that in the original analysis with hypertriglyceridemia, as in the current analysis the patients were evaluated years after the diagnosis of IgAGN. Use of ACE increased the statistical significance between the visits and it also seemed to be a prognostic variable in multivariate analysis, as did the amount of 24 -hour proteinuria, both being in line with previous publications $[10,19]$.

Taken together, the present data show that MetS is as prevalent in Finnish IgAGN patients as in the general population. It does not seem to be an independent prognostic variable in IgAGN. However, due to the small number of patients, our study may have lacked sufficient power to detect a significant effect of MetS on the progression of IgAGN.

\section{Disclosure Statement}

The authors have no conflicts of interest to declare.

\section{References}

1 Alberti KG, Eckel RH, Grundy SM, Zimmet PZ, Cleeman JI, Donato KA, Fruchart JC, James PT, Loria CM, Smith SC: Harmonizing the metabolic syndrome: a joint interim statement of the International Diabetes Federation Task Force on Epidemiology and Prevention; National Heart, Lung, and Blood Institute; American Heart Association; World Heart Federation; International Atherosclerosis Society; and International Association for the Study of Obesity. Circulation 2009;120:1640-1645.

-2 Johnson DW, Armstrong K, Campbell SB, Mudge DW, Hawley CM, Coombes JS, Prins JB, Isbel NM: Metabolic syndrome in severe chronic kidney disease: prevalence, predictors, prognostic significance and effects of risk factor modification. Nephrology (Carlton) 2007;12:391-398. 
Kaartinen et al.: Metabolic Syndrome in IgA Glomerulonephritis

- Kishikawa H, Nishimura K, Kato T, Kobayashi Y, Arichi N, Okuno A, Fujii N, Kyo M, Takahara S, Ichikawa Y: Prevalence of the metabolic syndrome in kidney transplantation. Transplant Proc 2009;41:181-183.

4 Ozdemir FN, Karakan S, Akgul A, Haberal M: Metabolic syndrome is related to long-term graft function in renal transplant recipients. Transplant Proc 2009;41:2808-2810.

5 Pietrzak-Nowacka M, Safranow K, Byra E, Binczak-Kuleta A, Ciechanowicz A, Ciechanowski K: Metabolic syndrome components in patients with autosomal-dominant polycystic kidney disease. Kidney Blood Press Res 2009;32:405-410.

6 Kurella M, Lo JC, Chertow GM: Metabolic syndrome and the risk for chronic kidney disease among nondiabetic adults. J Am Soc Nephrol 2005;16:2134-2140.

7 Lucove J, Vupputuri S, Heiss G, North K, Russell M: Metabolic syndrome and the development of CKD in American Indians: the Strong Heart Study. Am J Kidney Dis 2008;51:21-28.

-8 Luk AO, Ma RC, So WY, Yang XL, Kong AP, Ozaki R, Ko GT, Chow CC, Cockram CS, Chan JC, Tong PC: The NCEPATPII but not the IDF criteria for the metabolic syndrome identify type 2 diabetic patients at increased risk of chronic kidney disease. Diabet Med 2008;25:1419-1425.

-9 Servais A, Giral P, Bernard M, Bruckert E, Deray G, Isnard Bagnis C: Is serum cystatin-C a reliable marker for metabolic syndrome? Am J Med 2008;121:426-432.

10 Berthoux FC, Mohey H, Afiani A: Natural history of primary IgA nephropathy. Semin Nephrol 2008;28:4-9.

11 Kaartinen K, Syrjänen J, Pörsti I, Harmoinen A, Pasternack A, Huhtala H, Niemelä O, Mustonen J: Insulin resistance and the progression of IgA glomerulonephritis. Nephrol Dial Transplant 2007;22:778-783.

12 Wang L, Zhang Y, Chen S, Chen J, Zhuang Y, Chen J: Association of metabolic syndrome and IgA nephropathy. J Clin Pathol 2010;63:697-701.

13 Syrjänen J, Mustonen J, Pasternack A: Hypertriglyceridaemia and hyperuricaemia are risk factors for progression of IgA nephropathy. Nephrol Dial Transplant 2000;15:34-42.

$\checkmark 14$ Myllymäki J, Syrjänen J, Helin H, Pasternack A, Kattainen A, Mustonen J: Vascular diseases and their risk factors in IgA nephropathy. Nephrol Dial Transplant 2006;21:1876-1882.

15 Levey AS, Bosch JP, Lewis JB, Greene T, Rogers N, Roth D: A more accurate method to estimate glomerular filtration rate from serum creatinine: a new prediction equation. Modification of Diet in Renal Disease Study Group. Ann Intern Med 1999;130:461-470.

16 Pajunen P, Rissanen H, Härkänen T, Jula A, Reunanen A, Salomaa V: The metabolic syndrome as a predictor of incident diabetes and cardiovascular events in the Health 2000 Study. Diabetes Metab 2010;36:395-401.

17 Hall JE, Kuo JJ, da Silva AA, de Paula RB, Liu J, Tallam L: Obesity-associated hypertension and kidney disease. Curr Opin Nephrol Hypertens 2003;12:195-200.

18 Ritz E: Metabolic syndrome and kidney disease. Blood Purif 2008;26:59-62.

19 Barratt J, Feehally J: Treatment of IgA nephropathy. Kidney Int 2006;69:1934-1938. 